\title{
Aplicación del Modelo de Bohart y Adams en la Remoción de Mercurio de Drenajes de Minería por Adsorción con Carbón Activado
}

\author{
Hugo A. Rojas ${ }^{(1)}$, Diana C. Guerrero ${ }^{(1) *}$, Olga Y. Vásquez ${ }^{(2)}$ y Jesús S. Valencia ${ }^{(3)}$ \\ (1) Universidad Pedagógica y Tecnológica de Colombia, Grupo de Catálisis, Escuela de Química, \\ Facultad de Ciencias, Av. Norte, Tunja-Colombia. (e-mail: dianacga@msn.com) \\ (2) Universidad Central, Grupo del Agua y Desarrollo Sostenible, Carrera 5, № 21-38, \\ Bogotá, D.C.-Colombia. \\ (3) Universidad Nacional de Colombia, Laboratorio de Catálisis Heterogénea, Depto. de Química, \\ Facultad de Ciencias, Bogotá D.C., Colombia.
}

Recibido Ago. 17, 2011; Aceptado Oct. 11, 2011; Versión final recibida Dic. 08,2011

\section{Resumen}

Se aplica el modelo de Bohart y Adams al proceso de remoción de mercurio de los drenajes de minería por adsorción con carbón activado en columnas de lecho fijo. Para ello se analizaron diferentes drenajes de minas de carbón de Guachetá en Colombia y se simularon en el laboratorio las condiciones reales de vertido de los drenajes. Establecidas las condiciones de funcionamiento óptimo de la columna, se realizó un estudio detallado de curvas de ruptura, a través del modelo de Bohart y Adams, para determinar la vida útil del material adsorbente y la efectividad del método para la remoción del ion metálico. Se estableció que el tiempo de ruptura es de 20 min. y que la columna con el mejor rendimiento tiene $1.7 \mathrm{~cm}$ de diámetro y $2 \mathrm{~cm}$ de altura del lecho, bajo las condiciones de estudio. El modelo usado describió la parte inicial de la curva de ruptura y los parámetros obtenidos permitieron diseñar y optimizar el funcionamiento de sistemas de adsorción en lecho fijo para la remoción de iones $\mathrm{Hg}^{2+}$ de efluentes acuosos.

\section{Application of the Bohart and Adams Model in the Removal of Mercury from Mine Drainage by Adsorption with Activated Carbon}

\begin{abstract}
The model of Bohart and Adams was applied to the process of removal of mercury from mine drainage by adsorption with activated carbon in fixed bed columns. For this several drains from coal mines of Guachetá in Colombia, were studied. Once the optimum operating conditions of the column are established, a detailed study of breakthrough curves using the Bohart and Adams model was done, to determine the lifetime of the adsorbent material and the effectiveness of the method for removal the metal ions. It was established that the breakthrough time was $20 \mathrm{~min}$. and the column with the best performance was $1.7 \mathrm{~cm}$ in diameter and $2 \mathrm{~cm}$ bed length, under the study conditions. The model described the initial part of the breakthrough curve and the parameters of the model allow designing and optimizing the performance of fixed bed adsorption systems for the removal of $\mathrm{Hg}^{2+}$ ions fro aqueous mine wastes.
\end{abstract}

Keywords: mine drainage, mercury, activated carbon, breakthrough curves, Bohart and Adams model. 


\section{INTRODUCCIÓN}

Según la Organización Mundial de la Salud, los metales tóxicos que representan un mayor riesgo para el medio ambiente y la salud de los seres vivos son $\mathrm{Al}, \mathrm{Cr}, \mathrm{Mg}, \mathrm{Fe}, \mathrm{Co}, \mathrm{Ni}, \mathrm{Cu}, \mathrm{Zn}, \mathrm{Cd}, \mathrm{Hg}$ y $\mathrm{Pb}$, debido a que no se degradan en productos inocuos sino que tienden a acumularse en tejidos y órganos, causando diversas enfermedades y trastornos (Park et al., 2007). El mercurio se ha convertido en uno de los elementos más peligrosos pues su concentración en el medio ambiente ha aumentado considerablemente y está alcanzando niveles ciertamente tóxicos para muchos organismos, afecta el sistema nervioso central, degrada el ADN y tiende a acumularse y a magnificarse a través de la cadena trófica (Holmes et al., 2009). Las principales industrias responsables de la dispersión del mercurio son las de pintura, papel, aceite de refinación, procesamiento eléctrico, caucho, fertilizantes y carbón, aunque éste último en pequeñas cantidades. En Colombia, la mayor contaminación con este elemento se presenta en la minería aurífera, pues se estima que la cantidad liberada al ambiente, en este tipo de actividad está entre 80 y 100 toneladas al año y por ello existe una gran cantidad de zonas altamente afectadas que requieren ser reparadas (Vidal et al., 2010).

La minería a cielo abierto y subterráneo puede acelerar la producción de ciertos drenajes, que aunque en el suelo son naturales, la contribución minera hace que se caractericen por su bajo pH, elevadas concentraciones de hierro, sulfatos y metales pesados de composición variable, cuya carga depende del tipo de depósito de los minerales originarios. Estos efluentes pueden tratarse con productos químicos como cal y carbonato de sodio, lo que resulta en la producción de lodos voluminosos, que representan otro problema ambiental y costos adicionales (Sheroan et al., 2006). En Colombia, hay evidencia de la existencia de grandes cantidades de complejos mineros de explotación de carbón, donde los drenajes se vierten de manera indiscriminada. La Corporación Autónoma Regional (CAR) de Cundinamarca (Colombia), estima que las minas de carbón subterráneo vierten mensualmente cerca de $70.400 \mathrm{~m}^{3}$ de drenajes al recurso hídrico de la zona aledaña (Ministerio de Minas y Energía, 2007). Lugo, la preocupación por eliminar la mayor cantidad de contaminantes ha llevado al desarrollo de diferentes técnicas y métodos que incluyen: precipitación química, intercambio iónico, evaporación y concentración, ósmosis inversa, electrólisis, filtración y procesos de adsorción (Inbaraj et al., 2006).

La adsorción es un proceso de separación mediante el cual ciertos componentes en fase fluida se transfieren hacia un sustrato sólido, quedando física o químicamente enlazados a la superficie del adsorbente (Rivas et al., 2006). El carbón activado se utiliza en procesos de adsorción debido a su elevada área superficial, alta capacidad de adsorción, estructura porosa y eficiencia (Moreno et al., 2007). El carbón activado se produce, por métodos físicos o químicos, a partir de muchos precursores sólidos carbonosos (Bastidas et al., 2010). Este material puede emplearse en sistemas de lecho fijo, que consisten en una columna donde el adsorbente se deposita en su interior como un lecho y el líquido atraviesa la columna en sentido ascendente, horizontal o descendente. En el tratamiento de aguas residuales, el líquido fluye y los contaminantes se separan gradualmente (Zhang et al., 2010). El movimiento progresivo de la zona de adsorción puede representarse mediante las denominadas "curvas de ruptura" donde se determinan los requerimientos de tamaño del sistema, tiempo de contacto y velocidad de flujo; la operación puede detenerse cuando la concentración del adsorbible alcanza un valor típico del $10 \%$ de la concentración inicial; se obtiene así un "punto de ruptura" (Cespedes et al., 2007).

Para evaluar la adsorción dinámica de distintas sustancias (gaseosas y en disolución) se han propuesto y desarrollado numerosos modelos empíricos y uno de ellos, ideado para interpretar la remoción de cloro de corrientes de aire con cartuchos de carbón activado, es el de Bohart y Adams. En este modelo se asume que la velocidad de adsorción es proporcional a la capacidad de adsorción del carbón activado granular (GAC) y a la concentración del adsorbible. Por si solo, el modelo de Bohart y Adams proporciona un acercamiento simple y comprensivo al funcionamiento y evaluación de los sistemas de remoción en lecho fijo (columnas); por ello se usa en la descripción describir la parte inicial de la curva de ruptura (López et al., 2011). En su forma típica la ecuación de Bohart y Adams tiene la siguiente presentación: 


$$
\ln \left[\left(\frac{C_{o}}{C_{e}}\right)-1\right]=\ln \left(e^{\frac{k N o Z}{u}}-1\right)-k C_{o} t
$$

Donde $k$ es la constante de velocidad de adsorción, $N_{o}$ la capacidad de adsorción, $Z$ la altura del lecho fijo del adsorbible, $u$ es la velocidad de flujo lineal, $t$ es el tiempo de servicio de la columna $y$, $C_{o}$ y $C$ son, respectivamente, la concentración del adsorbible a la entrada al lecho y, a la salida de la columna, a un tiempo $t$.

La literatura abierta contiene numerosos estudios en los cuales se evalúa la capacidad de adsorción y el tiempo de servicio de columnas de carbón activado, procedente de diferentes precursores, dispuesto en forma de gránulos (GAC), fibras (ACF), monolitos o polvo (PAC). Así, por ejemplo, con esta metodología se examinó la remoción de colorante negro (RB5) con columnas de GAC obtenido de residuos de bambú (Bambusoideae); las concentraciones de colorante variaron entre 50 y $200 \mathrm{mg} \mathrm{L}^{-1}$, la velocidad de flujo se modificó entre 10 y $30 \mathrm{~cm}^{3} \mathrm{~min}^{-1}$ y la altura del lecho se cambió entre 40 y $80 \mathrm{~mm}$; el lecho con mayor capacidad de adsorción, equivalente a $39,02 \mathrm{mg} \mathrm{g}^{-1}$ se obtuvo con una concentración inicial de $100 \mathrm{mg} \mathrm{L}^{-1}, 80 \mathrm{~mm}$ de altura del lecho y un caudal $\mathrm{cm}^{3} \mathrm{~min}^{-1}$, con lo cual se demostró que a mayor cantidad de carbón activado existe mayor capacidad de remoción (Ahmad et al., 2009). En otros contextos, también se ha evaluado el potencial y la eficacia del carbón activado granular (GAC) para eliminar metales pesados en disolución, especialmente cadmio $(\mathrm{Cd})$ y plomo $(\mathrm{Pb})$, a través de la adsorción desde disoluciones modelo. Las curvas de ruptura y el modelo de Bohart y Adams se ha utilizado con éxito para predecir el grado de avance de la remoción y obtener la capacidad de adsorción; los resultados ponen que a través de diferentes pruebas en columnas la ecuación de Bohart y Adams se adapta muy bien al proceso e interpreta con éxito la tendencia observada en la remoción de cadmio y plomo (Jusoh et al., 2007). En otros estudios, las columnas de carbón activado se han utilizado para remover níquel $(\mathrm{Ni})$ y cobre $(\mathrm{Cu})$ de medios acuosos contaminados con aceite de palma (Yang et al., 2006); en ellos, se verifica que el proceso de remoción de cobre (Cu) se puede ajustar a tres modelos: Bohart y Adams, Thomas y Yoon-Nelson, no así el de níquel (Ni). En general, es posible verificar que el modelo de Bohart y Adams, que aunque fue desarrollado para gases, se ajusta muy bien para la remoción de tintas y colorantes de efluentes acuosos (Balci et al., 2011)

El presente estudio tiene como propósito evaluar, mediante curvas de ruptura, la capacidad de adsorción y el tiempo de servicio de un carbón activado granular (GAC), obtenido por carbonización y activación física de cáscara de coco (Cocos nucifera), frente a la remoción de iones mercurio $\left(\mathrm{Hg}^{2+}\right)$, presente en disoluciones modelo y en aguas de drenaje de minas de carbón establecidas en la región de Guachetá (Colombia).

\section{METODOLOGÍA}

\section{Caracterización del carbón activado}

La textura (área superficial y porosidad), la estructura y la morfología del carbón activado granular (GAC) de tipo comercial, obtenido por carbonización y activación de cuesco de coco (Cocos nucifera), se realizó mediante fisisorción de nitrógeno a $77 \mathrm{~K}$ (en un analizador de área superficial y porosidad Micromeritcs 2020), difracción de rayos X (en un difractómetro PanAlytical Pro MPD, en la modalidad Bragg-Brentano, utilizando la línea CuK con $\alpha=1,5418$ Å) y microscopía electrónica de barrido en un equipo JEOL5600-LV dotado con una microsonda para análisis EDS.

\section{Caracterización de los drenajes de minería}

Para la recolección y preservación de las muestras acuosas se siguió el método recomendado por el Instituto de Hidrología, Meteorología y Estudios Ambientales de Colombia (IDEAM) y por los métodos estandarizados para el análisis de agua y aguas residuales. El pH y la conductividad de cada muestra se determinaron con un equipo multiparámetro Hanna $\mathrm{HI}$ 9828; la acidez, la alcalinidad y el contenido de cloruros se establecieron con un analizador TitroLine Easy; el 
contenido de sulfatos se cuantificó mediante espectrofotometría UV-Vis, en un equipo Genesys 20. Finalmente, el contenido de $\mathrm{Fe}, \mathrm{Ca}, \mathrm{Mg}, \mathrm{Na}, \mathrm{K}, \mathrm{Al}, \mathrm{Mn}, \mathrm{Zn}$ y $\mathrm{Hg}$, se cuantificó mediante espectrofotometría de absorción atómica, en un equipo Varian AA240.

\section{Ensayos de remoción}

La disolución patrón (1000 $\mathrm{mg} \mathrm{L}^{-1}$ en $\mathrm{Hg}$ ), preparada a partir de $\mathrm{HgCl}_{2}$ (99,99\%), se obtuvo de Merck); a partir de esta disolución y por dilución con agua desionizada se obtuvieron concentraciones de 5 y $10 \mathrm{mg} \mathrm{L}^{-1}$. Para efectos de todos los ensayos, el $\mathrm{pH}$ final de las disoluciones se ajustó a un valor de 2,5 con la ayuda de una disolución $0,2 \mathrm{M}$ de $\mathrm{HNO}_{3}$ (de Merck). Con el fin de realizar las pruebas de remoción, la columna (construida en vidrio Pyrex®) se dispuso en forma vertical; el lecho de carbón (pesado con precisión a 0,1 $\mathrm{mg}$ ) se colocó sobre un tabique poroso ajustado al interior de la columna y para evitar el arrastre de las partículas de carbón, sobre el lecho se puso un retenedor de lana de vidrio desengrasada. La disolución se bombeó por la parte inferior de la columna con la ayuda de una bomba peristáltica (Masterflex®), a caudales fijo y modificables entre 1 y $10 \mathrm{~cm}^{3} \mathrm{~min}^{-1}$. En distintos experimentos, el diámetro interno de las columnas se varió entre 1,0 y $1,7 \mathrm{~cm}$; de igual forma, la altura del lecho $(Z)$ de carbón activado se modificó entre 1 y $2 \mathrm{~cm}$. Luego de realizar varios ensayos con las disoluciones modelo, se hicieron pruebas de remoción con aguas de drenaje real.

\section{RESULTADOS Y DISCUSIÓN}

\section{Caracterización del carbón activado}

En la Tabla 1 se muestran los resultados del análisis textural del carbón activado granular (GAC) utilizado en este trabajo, antes y después del proceso de adsorción de iones mercurio en disoluciones modelo. Se observa que las propiedades de área superficial, volumen de poro y tamaño promedio de poro no varían considerablemente por efecto de la remoción del mercurio. A pesar de que se trata de un material microporoso, el área superficial que posee el carbón utilizado en este trabajo es relativamente baja con relación al tamaño de poro. Al tratarse de un adsorbente comercial, no es posible, más allá de los análisis próximo y último inferir sobre la calidad del carbón activado con atención al proceso de activación. Algunos autores como Su et al. $\left(663 \mathrm{~m}^{2}\right.$ $\left.\mathrm{g}^{-1}\right)$, Achaw et al. $\left(524 \mathrm{~m}^{2} \mathrm{~g}^{-1}\right)$, Kunbin Yang et al. $\left(702 \mathrm{~m}^{2} \mathrm{~g}^{-1}\right)$, Singh et al. $\left(378 \mathrm{~m}^{2} \mathrm{~g}^{-1}\right)$ y Wei Su et al. (354 $\left.\mathrm{m}^{2} \mathrm{~g}^{-1}\right)$, citados por ( $\mathrm{Li}$ et al., 2008), han reportado áreas superficiales para carbones activados (obtenidos de cuesco de coco) que son comparables con las del adsorbente utilizado en esta investigación; sin embargo, los datos obtenidos por otros investigadores como Wei Li et al.

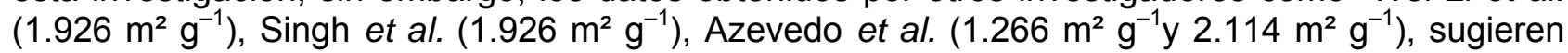
que es factible, en función de los procesos de carbonización y de los métodos de activación, desarrollar áreas superficiales mucho mayores.

Tabla 1. Propiedades de textura del carbón activado.

$\left.\begin{array}{|l|c|c|c|}\hline \multicolumn{1}{|c|}{\text { GAC }} & \begin{array}{c}\text { Área superficial Langmuir }\left(\mathrm{m}^{2}\right. \\ \left.\mathrm{g}^{-1}\right)\end{array} & \begin{array}{c}\text { Volumen de poro } \\ \left(\mathrm{cm}^{3} \mathrm{~g}^{-1}\right)\end{array} & \text { Tamaño de poro }(\AA \\ )\end{array}\right)$

En la Figura 1 se muestran los perfiles de difracción de rayos $X$ obtenidos de las muestras de carbón activado, fresco y usado en los procesos de adsorción de iones mercurio. Todos los patrones de difracción de rayos $X$ son compatibles con una estructura predominantemente amorfa, lo cual significa que las partículas de grafeno no son lo suficientemente grandes para responder apropiadamente al fenómeno de dispersión. Sin embargo, es posible observar algunas reflexiones en las posiciones $2 \theta$ de $26^{\circ}$ y $43^{\circ}$ que sugieren cierta cristalinidad como resultado de una alineación de las capas (de grafeno); el grupo de Tongpoothorn ha encontrado resultados 
similares al evaluar la estructura de carbones activados mediante DRX (Tongpoothorn et al., 2011). De otra parte, los espectros de difracción muestran, hasta donde alcanza la sensibilidad de la técnica, que la adsorción de iones mercurio no genera cambios drásticos en la estructura del carbón activado.

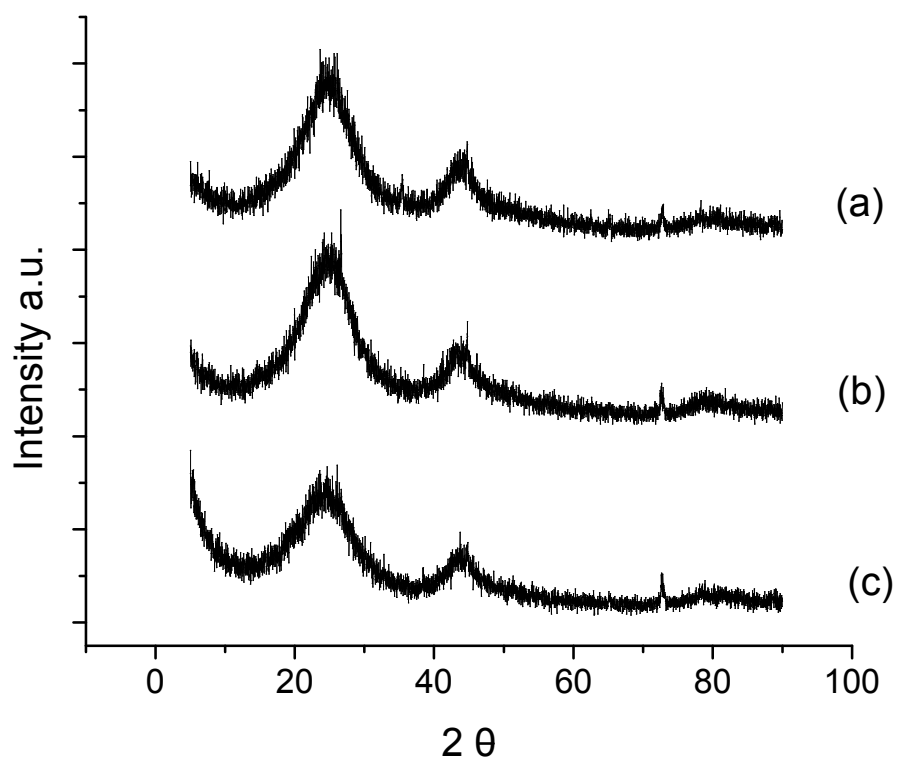

Fig. 1: Patrones de difracción de rayos-X del carbón activado. (a) Antes del proceso de adsorción. (b) y (c) Carbones activados empleados en adsorción de 5 ppm y 10 ppm, respectivamente.

Tal como se ilustra en la Figura 2, las imágenes de microscopía electrónica de barrido muestran un superficie con textura rugosa, relativamente mesoporosa, con estructura amorfa y aspecto heterogéneo. Las micrografías antes y después del proceso de adsorción (Figuras 2(a) y 2(b), permiten observar que al menos a este nivel de magnificación (tamaño micrométrico) el proceso de remoción de iones mercurio no modifica de manera significativa la superficie del material adsorbente, reafirmando los resultados obtenidos por difracción rayos $X$ y el estudio de propiedades de textura.

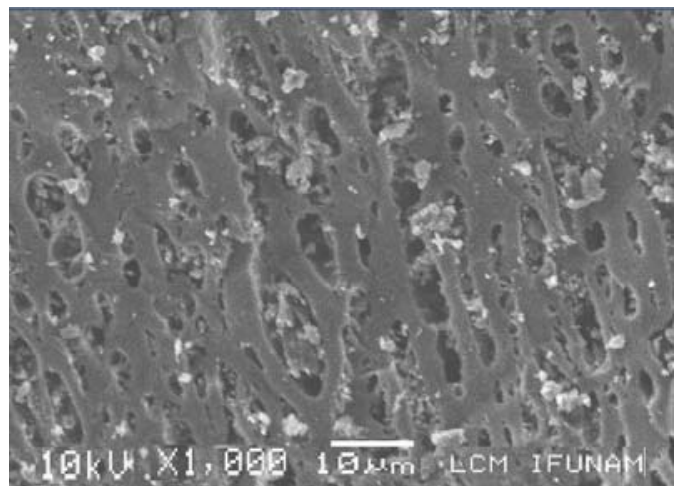

(a)

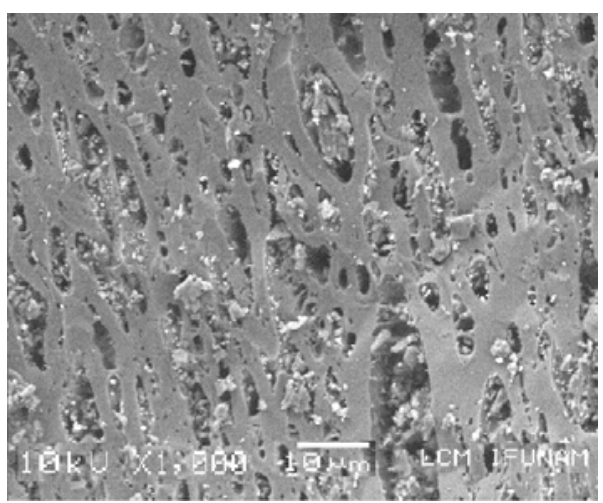

(b)

Fig. 2: Micrografías carbón activado. (a) Micrografía antes del proceso de adsorción. (b) Micrografía después del proceso de adsorción

La Figura 3(a) representa el espectro de fluorescencia, captado mediante microsonda EDX, obtenido para una muestra del carbón activado sin usar. En el material adsorbente es evidente la presencia de carbono (línea C1s, como era de esperarse) y de potasio; la existencia de potasio se atribuye a la naturaleza del precursor (cáscara de coco), situación que ha sido corroborada por otros investigadores (Raveendran et al., 1995). Debido a que no existen evidencias (trazas) de la presencia de elementos tipo $\mathrm{Zn}, \mathrm{Cl}, \mathrm{P}$ o $\mathrm{S}$, se presume que el material carbonoso de partida se activó por procesos físicos (vapor de agua o CO2) y que la superficie del adsorbente no se ha 
sometido a modificaciones adicionales. La Figura 3(b) muestra el espectro de fluorescencia de rayos $\mathrm{X}$ del carbón activado expuesto a la disolución de 5 ppm de $\mathrm{HgCl}$; es claro, por la línea La1 que aparece cerca de 1,2 keV.

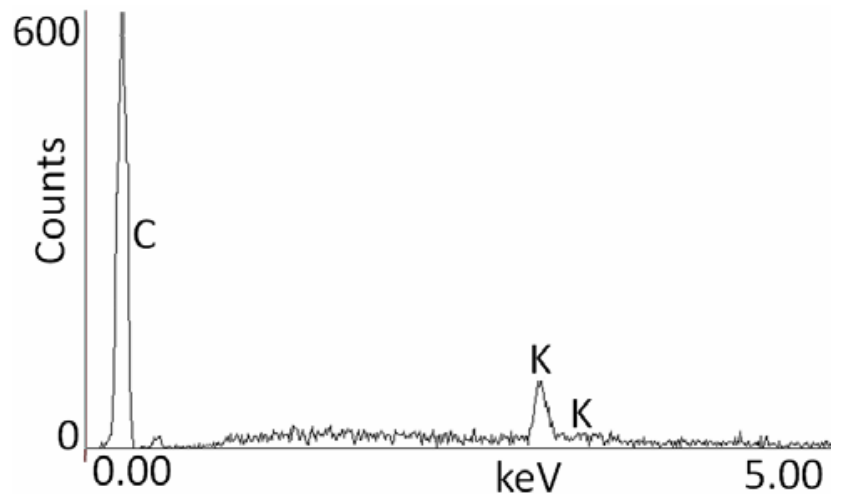

(a)

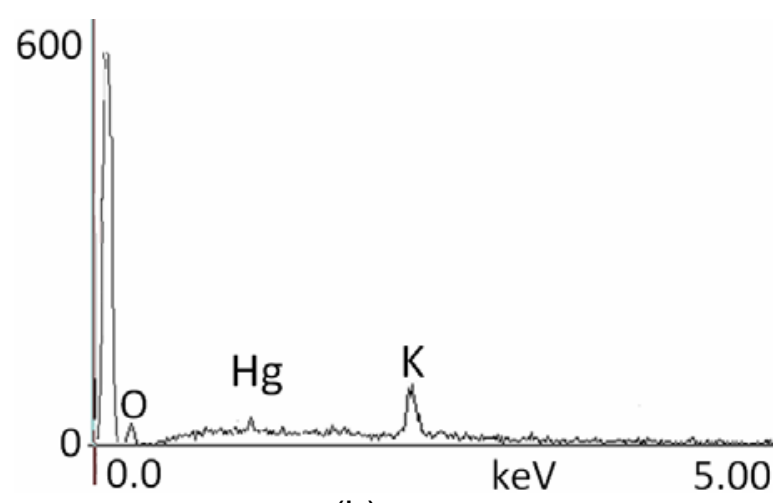

(b)

Fig. 3: Espectros XRF (vía EDX). (a) Muestra GAC fresca. (b) Muestra GAC usada con 5 ppm de $\mathrm{Hg}^{2+}$.

\section{Caracterización de los drenajes de minería}

En este trabajo se analizaron las aguas de drenaje de nueve minas (de socavón) de carbón, muestreadas en ocho oportunidades. En la Tabla 2 se muestran las convenciones utilizadas para identificar los drenajes y en las Figuras 4 a 7 se reúnen los diferentes parámetros fisicoquímicos establecidos para las distintas aguas de drenaje, incluida la composición química en términos de los elementos más abundantes.

Tabla 2. Convenciones de las aguas de drenaje estudiadas.

\begin{tabular}{|l|c|l|c|}
\hline \multicolumn{1}{|c|}{ Mina de procedencia } & Convención & \multicolumn{1}{c|}{ Mina de procedencia } & Convención \\
\hline El roble & $\mathrm{Ro}$ & La esperanza & $\mathrm{E}$ \\
\hline La virgen & $\mathrm{V}$ & Zuncho de gemela & $\mathrm{Z}$ \\
\hline El rubí del callejón & $\mathrm{RC}$ & Latincar & $\mathrm{L}$ \\
\hline El rinconcito & $\mathrm{Ri}$ & Carbocuality & $\mathrm{C}$ \\
\hline La mejía & $\mathrm{M}$ & & \\
\hline
\end{tabular}

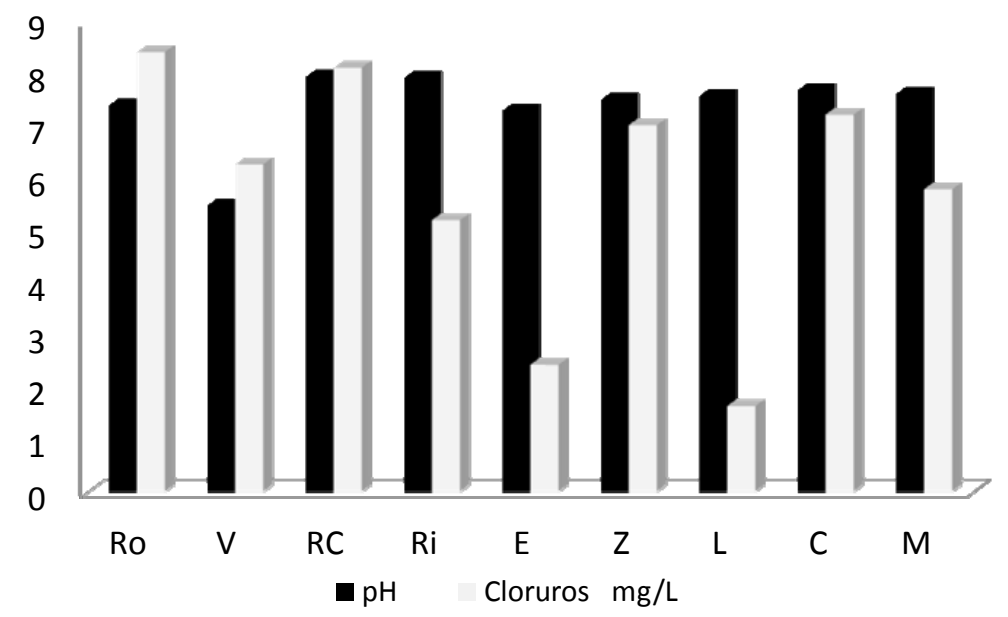

Fig. 4: $\mathrm{pH}$ y contenido de cloruros de aguas de drenaje de minería de carbón. 


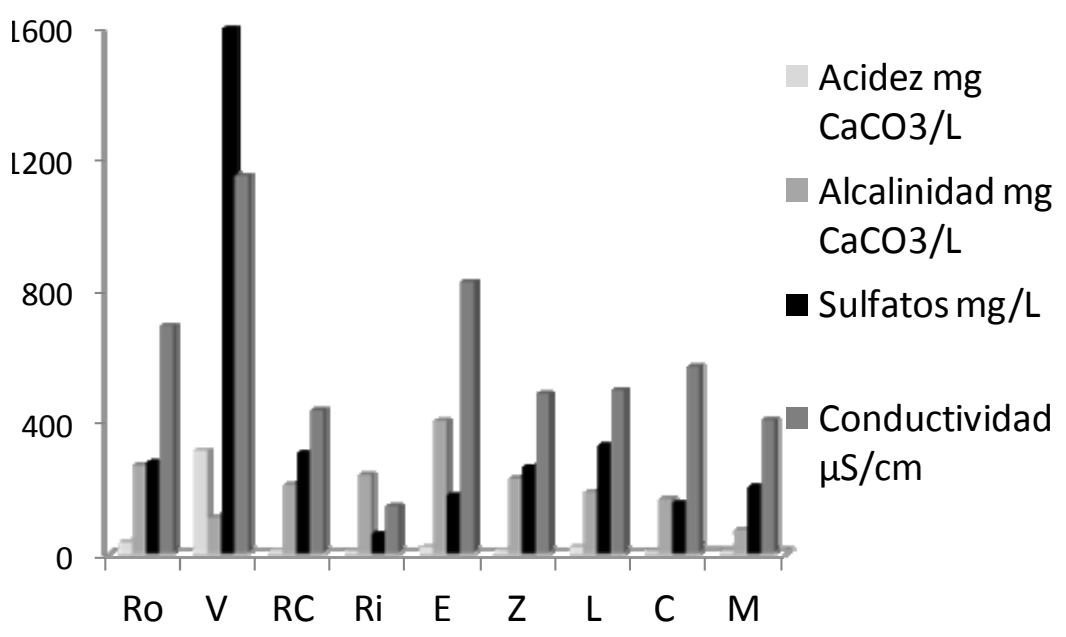

Fig. 5: Acidez, alcalinidad, sulfatos y conductividad de aguas de drenaje de minería de carbón.

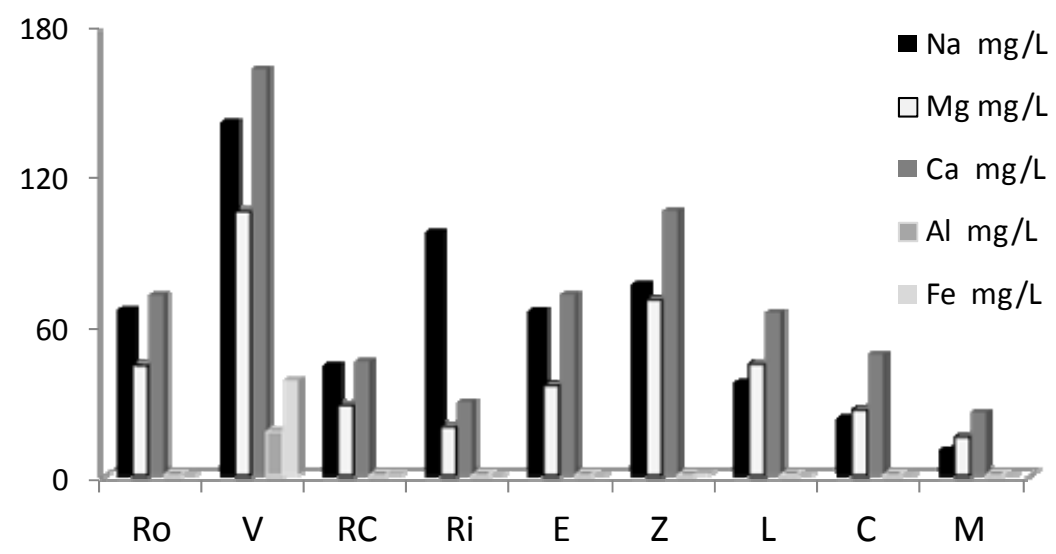

Fig. 6: Contenido de $\mathrm{Na}, \mathrm{Mg}, \mathrm{Ca}, \mathrm{Al}$ y Fe en aguas de drenaje de minería de carbón.

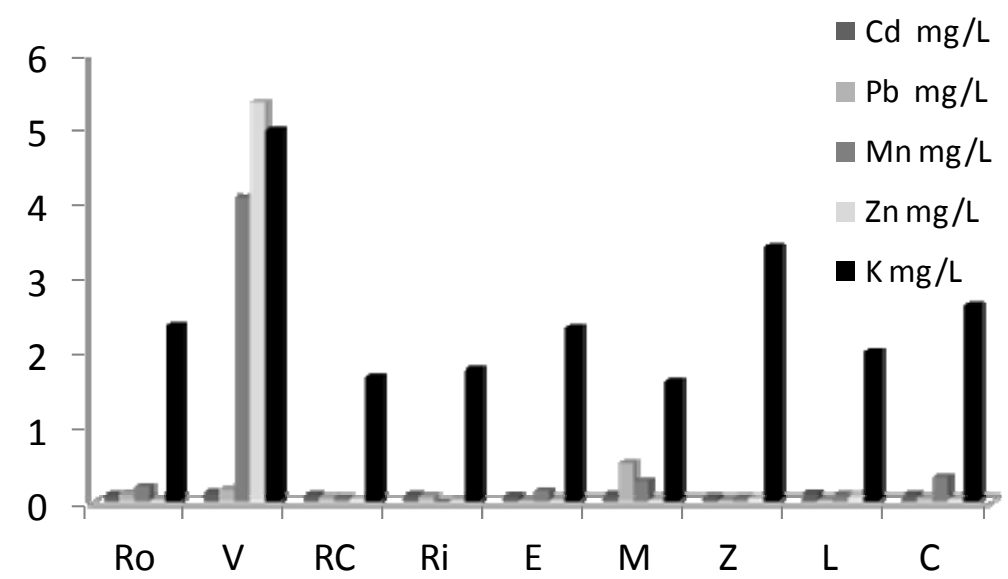

Fig. 7: Contenido de $\mathrm{Mn}, \mathrm{Zn}, \mathrm{Cd}, \mathrm{K}$ y Pb en aguas de drenaje de minería de carbón.

Los análisis fisicoquímicos de las aguas de drenaje de minería del carbón ponen en evidencia una variación del $\mathrm{pH}$, que está relacionada con los contenidos de iones sulfato y de hierro que se asocian a la oxidación de la pirita $\left(\mathrm{FeS}_{2}\right)$. Este proceso puede explicarse de la siguiente manera (Ríos et al., 2008):

$\mathrm{FeS}_{2(\mathrm{~s})}+3 \mathrm{O}_{2(g)}+3 \mathrm{H}_{2} \mathrm{O}_{(l)} \rightarrow \mathrm{Fe}^{2+}{ }_{(a c .)}+2 \mathrm{SO}_{4}{ }^{2-}{ }_{(a c .)}+2 \mathrm{H}_{3} \mathrm{O}_{(\text {ac. })}^{+}$

$4 \mathrm{Fe}^{2+}{ }_{(a c .)}+\mathrm{O}_{2(g)}+4 \mathrm{H}_{3} \mathrm{O}^{+} \rightarrow 4 \mathrm{Fe}^{3+}{ }_{(a c .)}+6 \mathrm{H}_{2} \mathrm{O}_{(l)}$ 


$$
\begin{aligned}
& \mathrm{Fe}^{3+}{ }_{(a c .)}+6 \mathrm{H}_{2} \mathrm{O}_{(l)} \rightarrow \mathrm{Fe}(\mathrm{OH})_{3(s)}+3 \mathrm{H}_{3} \mathrm{O}^{+}{ }_{(a c .)} \\
& \mathrm{FeS}_{2(s)}+14 \mathrm{Fe}^{3+}{ }_{(a c .)}+12 \mathrm{H}_{2} \mathrm{O}_{(l)} \rightarrow 15 \mathrm{Fe}^{2+}{ }_{(a c .)}+2 \mathrm{SO}_{4}{ }^{2-}{ }_{(a c .)}+8 \mathrm{H}_{3} \mathrm{O}^{+}{ }_{(a c .)}
\end{aligned}
$$

Las aguas de drenaje de la mina La virgen presentaron un carácter fuertemente ácido, elevada fuerza iónica y, valores extremos para el $\mathrm{pH}$, la acidez, la conductividad eléctrica y el contenido de cloruros; junto con estos parámetros, se registraron concentraciones muy altas de sulfatos e hierro, valores considerable de sodio y aluminio, lo cual reflejó una notable actividad frente a la capacidad para acelerar los procesos de hidrólisis de los minerales. Aún así y a pesar del contenido de cationes básicos como $\mathrm{Mg}^{2+}$ y $\mathrm{Ca}^{2+}$ no fue posible registrar efectos en la neutralización de la acidez del sistema. Los drenajes de las minas de carbón restantes fueron de carácter neutro y básico, una situación que sugiere que las superficies de los depósitos de los drenajes dentro de la mina son pizarras de carácter alcalino; en consecuencia, gran parte de la acidez generada durante la oxidación de la pirita resulta neutralizada, principalmente por la presencia $\mathrm{Ca}^{2+} \mathrm{y} \mathrm{Mg}^{2+}$.

De otro lado, en la mayoría de las aguas de drenaje los niveles de $\mathrm{Fe}^{3+}$ y $\mathrm{Al}^{3+}$ resultaron relativamente bajos, al igual que los contenidos de otros iones metálicos como $\mathrm{Mn}^{2+}, \mathrm{Na}^{+}, \mathrm{Zn}^{2+}$ y $\mathrm{K}^{+}$. Tal como se destaca en la Tabla 3 , en algunas aguas de drenaje y en un río aledaño (que recolecta estas aguas) fue posible detectar la presencia de mercurio que aunque en pequeña cantidad, destacan el hecho de que este peligroso metal puede estar asociado a la minería del carbón.

El contenido de mercurio en el drenaje de la mina Carbocuality es ligeramente superior al límite permitido por la legislación colombiana que establece un valor de 0,001 $\mathrm{mg} \mathrm{L}^{-1}$; sin embargo, en términos de la afectación de la cadena trófica y de la bioacumulación, esta información supone la existencia de un riesgo permanente para el medio ambiente y los pobladores de la zona. El hecho de que no exista mercurio en la mayoría de las aguas de drenaje objeto de este estudio puede depender de la misma actividad minera, de la convergencia de vertimientos, de fenómenos de lixiviación, de movimiento de aguas subterráneas, de la frecuencia de los procesos de lavado y de la presencia de residuos (cenizas y compuestos de azufre).

Tabla 3. Presencia de mercurio en aguas de drenaje de mineros minería del carbón

\begin{tabular}{|l|c|}
\hline \multicolumn{1}{|c|}{ Drenaje } & $\mathrm{Hg}\left(\mathrm{mg} \mathrm{L}^{-1}\right) \times 10^{-4}$ \\
\hline Efluente aledaño & 4,0 \\
\hline Carbocuality & 11 \\
\hline El roble & 4,0 \\
\hline El rubí del callejón & 4,0 \\
\hline
\end{tabular}

Proceso de remoción de iones $\mathrm{Hg}^{2+}$

La Figura 8 ilustra un conjunto de curvas de ruptura, obtenidas para un lecho de $2 \mathrm{~cm}$, con una concentración inicial $\left(C_{0}\right)$ de $5 \mathrm{mg} \mathrm{L}^{-1}$ de ion $\mathrm{Hg}^{2+}$. En todos los casos se observa la característica tendencia sigmoidea y en general, una rápida saturación del lecho, una situación que se traduce en tiempos de servicio $\left(t_{B}\right)$ relativamente cortos (no mayores a $20 \mathrm{~min}$ ). El desplazamiento hacia tiempos de servicio mayores se hace evidente en la medida en que se incrementa el diámetro de la columna ya que, una mayor cantidad (en masa) del adsorbible supone una mayor capacidad de adsorción. Un hecho interesante, que guarda relación con la baja capacidad de adsorción que tienen numerosos adsorbentes, incluido el carbón activado, frente a los metales pesados $(\mathrm{Pb}, \mathrm{Cd}$, $\mathrm{Sb}, \mathrm{Ni}$ ) es que el carbón activado se satura muy rápido y esto hace predecir el requerimiento de lechos muy altos o de tabiques muy gruesos para que al remover hasta el $90 \%$ de la concentración inicial de ión $\mathrm{Hg}^{2+}$ se alcance un de servicio realmente útil (horas) para su aplicación a eventuales situaciones prácticas. 


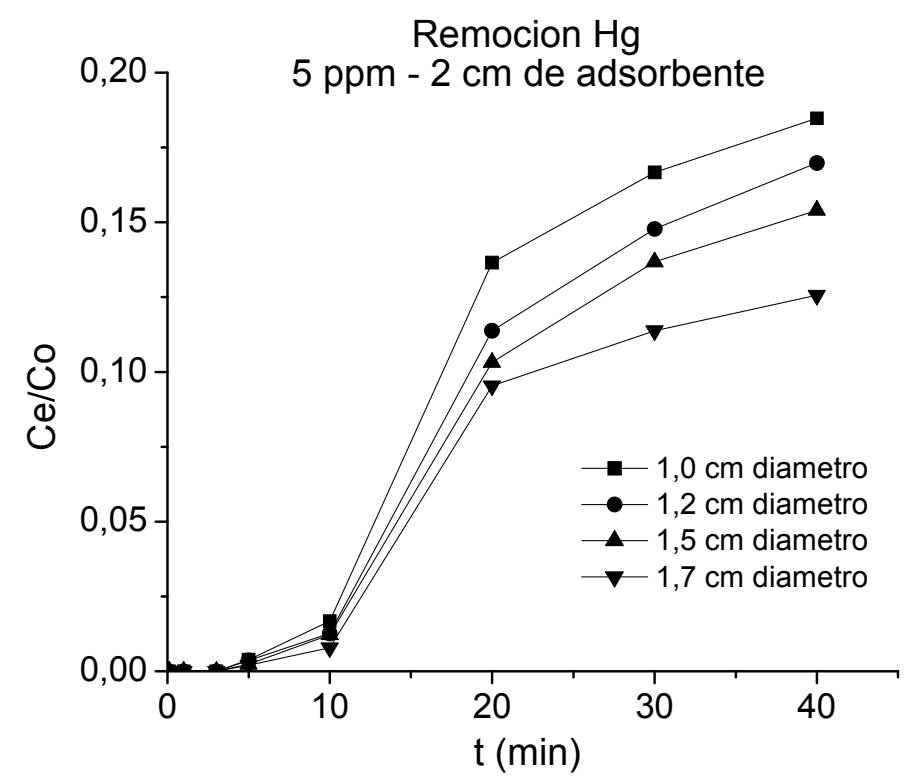

Fig. 8: Curvas de ruptura para la remoción de mercurio en disoluciones de 5 ppm.

En la Figura 9 se representan las curvas de ruptura obtenidas para la remoción de iones $\mathrm{Hg}^{2+}$ de una disolución de $10 \mathrm{mg} \mathrm{L}^{-1}$ adsorción. Aunque las tendencias, para una altura de lecho de $2 \mathrm{~cm}$, a idéntica velocidad de flujo $\left(10 \mathrm{~cm}^{3} \mathrm{~min}^{-1}\right)$, en función del diámetro de la columna, son sigmoideas, se destacan algunos hechos interesantes: 1. Los lechos de carbón se saturan más rápidamente con el aumento de la concentración. 2. Los tiempos de servicio de la columna son más pequeños con el incremento de la concentración. 3. El tiempo de servicio de la columna tiende a aumentar con el incremento del diámetro de la columna. Así, frente al carácter predictivo de las curvas de ruptura, se sigue que con el aumento de la concentración, para una misma carga de adsorbente, bajo idénticas condiciones de velocidad de flujo y de geometría de columna, disminuye el volumen de disolución tratado y por consiguiente el rendimiento del proceso (El Qada et al., 2008).

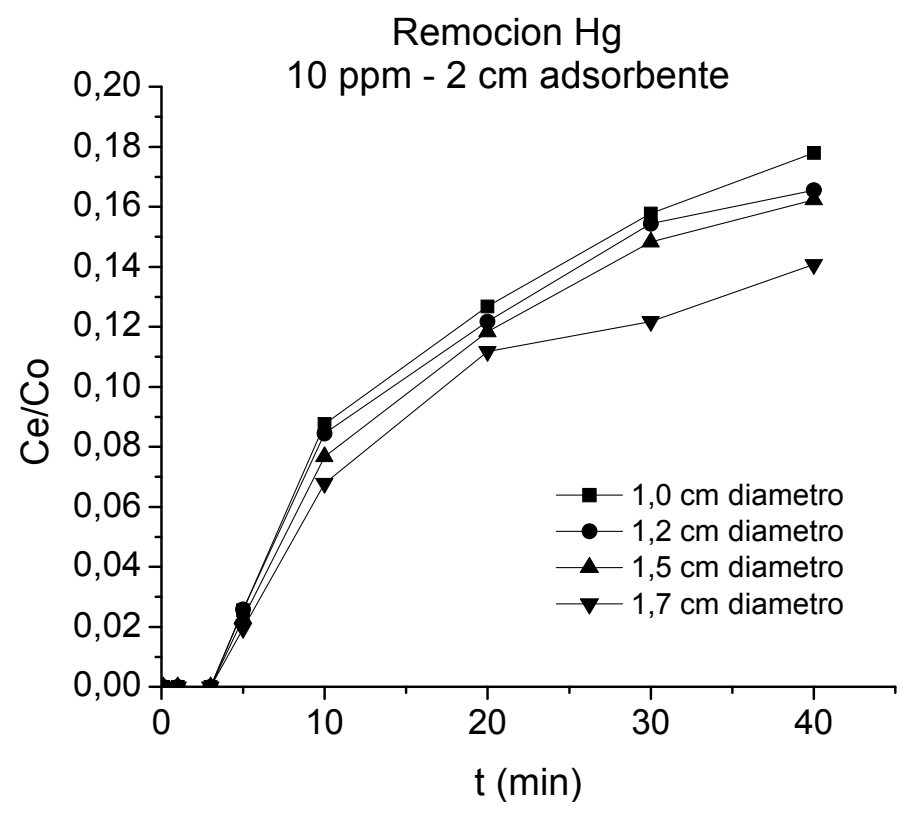

Fig. 9: Curvas de ruptura para la remoción de mercurio en disoluciones de 10 ppm.

La mayor remoción de iones $\mathrm{Hg}^{2+}$ en función del diámetro la columna (para una misma altura de lecho) se interpreta como un tiempo de servicio más prolongado de la columna debido al aumenta la cantidad de adsorbente y esto, en forma extensiva, implica mayor superficie para la adsorción, mayor números de grupos superficiales que gracias a un efecto ligando pueden formar compuestos de coordinación con los iones $\mathrm{Hg}^{2+}$, mayores posibilidades de retención a través de 
intercambio iónico y mayor cantidad de cenizas (óxidos metálicos) en el adsorbente que posiblemente contribuyan a incrementar la capacidad de remoción; de hecho, se admite que la remoción de iones metálicos en disolución, por parte del carbón activado, es un proceso complejo que en esencia nos solo depende de la naturaleza del ión metálico sino también de la naturaleza fisicoquímica del carbón.

En la Figura 10 se ilustra la curva de ruptura, obtenida para un drenaje real (Carbocuality), en una columna de $1,7 \mathrm{~cm}$ de diámetro, con una altura de lecho de $2 \mathrm{~cm}$ y una velocidad de flujo de 10 $\mathrm{cm}^{3} \mathrm{~min}^{-1}$, en contraste las disoluciones modelo. Allí, se destaca que el tiempo de servicio de la columna (evaluado a $C / C_{0}=0,1$ ) es de 50 min.

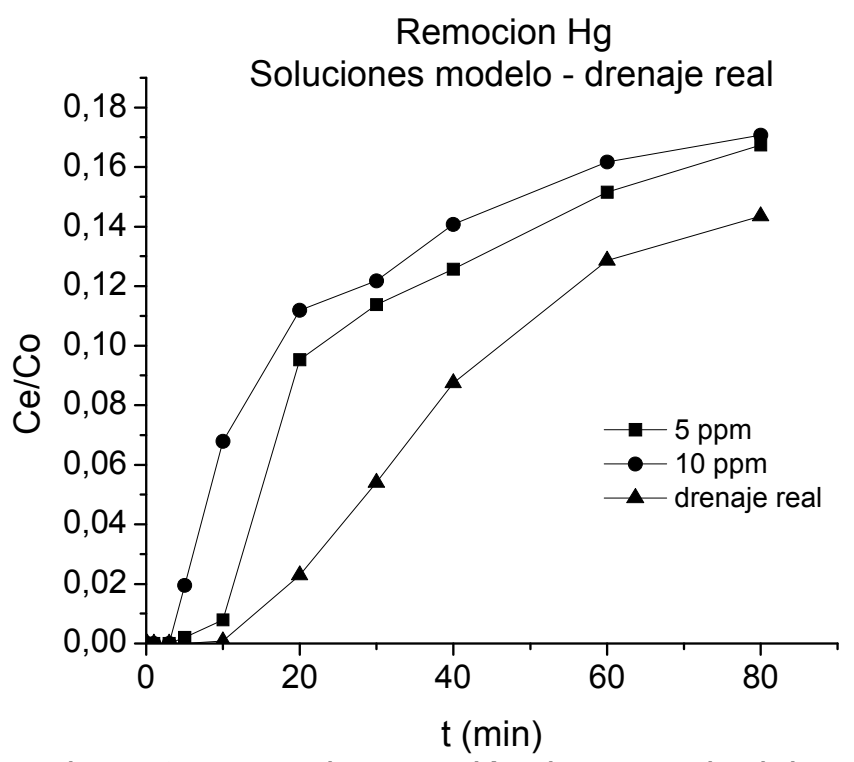

Fig. 10: Curva de ruptura para la remoción de mercurio del agua de un drenaje real de minería de carbón.

\section{Aplicación del modelo Bohart y Adams}

El modelo Bohart y Adams desempeña un papel fundamental para evaluar el comportamiento del carbón activado frente a los drenajes de minería permitiendo evidenciar que el uso de columnas de lecho fijo empleando carbón activado como adsorbente es un método efectivo en la remoción del mercurio. Experimentalmente, se estableció que el punto de ruptura del carbón activado empleado en los ensayos ocurre alrededor de 20 minutos, evidenciándose que la fase inicial del proceso de adsorción se adapta a la curva determinada teóricamente. Esta duración se incrementa levemente con el aumento de la altura del lecho, diámetro de la columna y por lo tanto con una mayor cantidad de carbón activado, debido a que un aumento del lecho implica una mayor superficie disponible (Meena et al., 2005) .

En la Figura 11 se expone una superposición de la curva de ruptura calculada a partir del modelo de Bohart y Adams y una curva experimental. A partir de este hecho se puede inferir que la tendencia descrita por el modelo y la curva experimental coinciden hasta tiempos muy cercanos al tiempo de ruptura $\left(t_{\mathrm{B}}, C / C_{0}=0,1\right)$; luego, esta aproximación sugiere que en términos predictivos los parámetros de la curva experimental, esto es, la constante de velocidad de adsorción ( $k$ ), la capacidad de adsorción $\left(N_{o}\right)$, la altura del lecho del adsorbible $(Z)$, permiten estimar (para una relación $C / C_{o}=0,1$ ) la vida útil de una columna (tiempo de servicio) para el tratamiento de un vertimiento real, que se desplaza (por un canal abierto o cerrado) con una velocidad lineal $u$.

En este trabajo, a pesar de que la remoción dinámica de iones $\mathrm{Hg}^{2+}$, presente en aguas de drenaje de minería de carbón es similar a la obtenida a escala de laboratorio con disoluciones modelo, corroborado así por el tiempo de utilidad de cada columna, conviene entonces caracterizarla en condiciones de operación reales pues, tal parece que el grado de ajuste con el modelo de Bohart y Adams depende de las características propias de cada drenaje. 


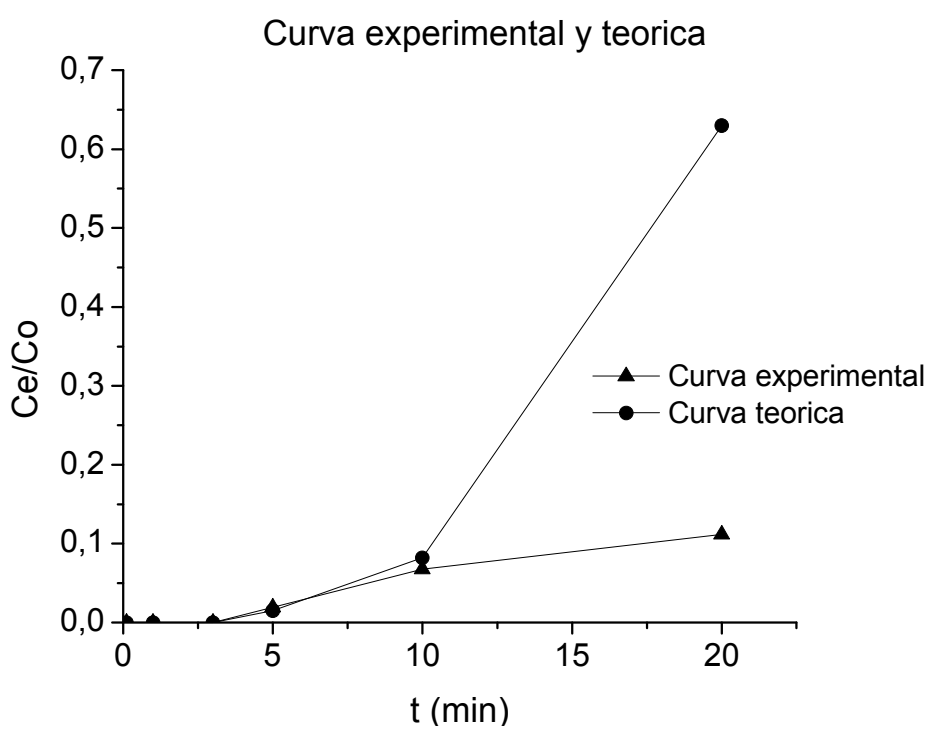

Fig. 11: Curva de ruptura modelada para el proceso de remoción de iones mercurio en disolución acuosa, sobre carbón activado.

\section{CONCLUSIONES}

En este trabajo se determinó que el carbón activado granular, procedente de la carbonización y activación de cuesco de coco, es un sólido adecuado para los procesos de remoción de iones $\mathrm{Hg}^{2+}$ presentes en aguas de drenajes de minería de carbón de la región de Guachetá (Colombia). La capacidad de remoción y la vida útil de las columnas dependen de las características del sólido (área superficial, tamaño de poro, grupos superficiales y capacidad de intercambio iónico), como también de la geometría de la columna, de la concentración del adsorbible, de la velocidad de flujo, de la altura del lecho del adsorbente y de la naturaleza misma del drenaje. Mediante técnicas analíticas del estado sólido (área superficial y porosidad, DRX, SEM y EDS) se evaluaron las características de textura, estructura, morfología y composición de un carbón activado, granular, de tipo comercial, que resultó con baja área superficial, amorfo y porosidad heterogénea. Frente al proceso de adsorción dinámico (en columnas), el modelo de Bohart y Adams describió la parte inicial $(10 \%)$ de la curva de ruptura, proporcionando parámetros cuantitativos (constante de velocidad de adsorción, capacidad de adsorción y altura del lecho) que permitieron, en forma predictiva, diseñar y optimizar el funcionamiento de sistemas de adsorción basados en lecho fijo para la remoción de iones $\mathrm{Hg}^{2+}$ de efluentes acuosos.

\section{AGRADECIMIENTOS}

Los autores agradecen a Colciencias por el apoyo financiero al proyecto con código 1225-45421803. A la DIN UPTC. A la Universidad Central por las facilidades para el desarrollo del trabajo. AI IF-UNAM por las facilidades en el estudio de microscopia realizado.

\section{REFERENCIAS}

Ahmad, A. A. Hameed, B. H., Reduction of COD and color of dyeing effluent from a cotton textile mill by adsorption onto bamboo-based activated carbon. Journal of Hazardous Materials (172) 1538-1543. (2009).

Balci, B., Keskinkan, O., Avci, M., Use of BDST and an ANN model for prediction of dye adsorption efficiency of Eucalyptus camaldulensis barks in fixed-bed system. Expert Systems with Applications (38). 949-956. (2011)

Bastidas, M., Buelvas, L., Márquez, M., Rodríguez, K., Producción de carbón activado a partir de precursores carbonosos del Departamento del Cesar, Colombia. Información Tecnológica (21). 87-96. (2010). 
Céspedes, N. E., Remoción de cromo vi de soluciones acuosas por adsorción sobre carbones activados modificados. Tesis de Maestría. Universidad Nacional de Colombia. Facultad de Ciencias. Departamento de Química. (2007).

El Qada, E., Allen, S., Walker, G., Adsorption of basic dyes from aqueous solution onto activated carbons. Chemical Engineering Journal (135) 174-184. (2008).

Holmes, P., James, K., Levy, L. S., Is low-level environmental mercury exposure of cancer to human health?. Science of the Total Enviroment (408), 171-182. (2009).

Inbaraj, B., Sulochana, N., Mercury adsorption on a carbon sorbent derived from fruit shell of Terminalia catappa. Journal of Hazardous Materials (B133), 283-290. (2006).

Jusoh, A., Shiung, L., A simulation study of the removal efficiency of granular activated carbon on cadmium and lead. Desalinitation (206). 9-16. (2007).

Li, W., Yang, K., Peng, J., Zhang, L., Guo, S., Xia, H., Effects of carbonization temperatures on characteristics of porosity in coconut shell chars and activated carbons derived from carbonized coconut shell chars. Industrial Crops and Products (28). 190-198. (2008).

Lopes, C.B., Pereira, E., Lin, Z., Fixed-bed removal of $\mathrm{Hg}^{2+}$ from contaminated water by microporous titanosilicate ETS-4: Experimental and theoretical breakthrough curves. Microporous and Mesoporous Materials (2011)

Meena, A., Mishra, G.K., Rai, P.K., Chitra, R., Nagar, P.N., Removal of heavy metal ions from aqueous solutions using carbon aerogel as an adsorbent. Journal of Hazardous Materials (B122).161-170. (2005)

Ministerio de Minas y Energía. Diseño de agenda para el desarrollo productivo y competitivo del distrito minero Zipa-Samacá. Bogotá. (2007)

Moreno, J., Navarrete, L., Giraldo, L., García, V., Adsorción de fenol y 3-clorofenol sobre carbones activados mediante calorimetría de inmersión. Información Tecnológica. 18(3). 71-80. (2007)

Park, H., Kim, T., Yun Chae, M., Yoo, I., Activated carbon-containing alginate adsorbent for the simultaneous removal of heavy metals and toxic organics. Process Biochemistry (42), 1371-1377. (2007)

Raveendran, K., Ganesh, A., Khilar, K.C., Influence of mineral matter on biomass pyrolysis characteristics. Fuel. (12). 1812-1822. (1995).

Rios, C.A., Williams, C.D., Roberts, C.L., Removal of heavy metals from acid mine drainage (AMD) using coal fly ash, natural clinker and synthetic zeolites. 2008. Journal of Hazardous Materials (156), 23-35. (2008).

Rivas, G., Caracterización de la biosorción de cromo con hueso de aceituna Universidad de Granada. Facultad de Ciencias. Departamento de Ingeniería Química. (2006)

Sheoran, A.S., Sheoran, V., Heavy metal removal mechanism of acid mine drainage in wetlands: A critical review. Minerals Engineering. (19), 105-116. (2006)

Tongpoothorn, W., Sriuttha, M., Homchan, P., Chanthai, S., Ruangviriyachai, C., Preparation of activated carbon derived from Jatropha curcas fruit shell by simple thermo-chemical activation and characterization of their physico-chemical properties. Chemical Engineering Research and Design (89), 335-340. (2011).

Vidal, J., Marrugo, J., Jaramillo, B., Perez, L., Remediación de suelos contaminados con mercurio utilizando guarumo (Cecropia peltata). Ingeniería y Desarrollo p. 114-115. (2010).

Yang, T., Lua, A.C., Textural and chemical properties of zinc chloride activated carbons prepared from pistachio-nut shells. Mater. Chemical Physics. (100), 438-444. (2006).

Zhang, C., Zhang, R., Xing, B., Cheng, G., Effect of pore structure on the electrochemical performance of coal-based activated carbons in non-aqueous electrolyte. New Carbon Materials (25), 129-133. (2010). 